\title{
Latest Results from the Daya Bay Reactor Neutrino Experiment
}

\author{
Shengchao $\mathbf{L i}^{*}$ \\ (on behalf of the Daya Bay Collaboration) \\ Center for Neutrino Physics \\ Department of Physics \\ Virginia Tech, Blacksburg, VA \\ E-mail: scli@vt.edu
}

Starting in 2011, the Daya Bay Reactor Neutrino Experiment has been observing antineutrinos from six nuclear reactors with eight identically designed underground antineutrino detectors in three experimental halls. It has accumulated the world's largest dataset of electron antineutrino candidates. The measurement of the neutrino mixing angle $\theta_{13}$ and the neutrino mass squared difference $\left|\Delta m_{e e}^{2}\right|$ have reached a precision of better than $4 \%$. The large dataset allows study of a variety of topics in neutrino physics, such as absolute reactor flux and spectrum. In this poster, we present the latest results from Daya Bay on several topics.

The 20th International Workshop on Neutrinos (NuFact2018)

12-18 August 2018

Blacksburg, Virginia

* Speaker. 


\section{Introduction}

The Daya Bay Reactor Neutrino Experiment studies antineutrinos from six reactors near Shenzhen, China, with eight identically designed antineutrino detectors. Four of the detectors are split between two near halls and the other four are placed in the far hall. The near detectors constrain the reactor antineutrino flux; the far detectors measure antineutrino disappearance. The six reactor cores in Daya Bay produce $17.4 \mathrm{GW}_{\text {th }}$ power, producing an estimated $35 \times 10^{20}$ neutrinos per second. Each antineutrino detector (AD) has three cylindrical zones [1], starting for the center: a 20-ton Gd-doped liquid scintillator, a 22-ton liquid scintillator and a 40-ton mineral oil buffer. The energy resolution [1] in the detectors is $8.5 \% / \sqrt{E}(\mathrm{MeV})$. Outside the detectors, water Cherenkov detector is used to shield the ADs from natural radioactivity and to detect cosmogenic muons; together with resistive plate chambers placed on top of the detector, it produces an efficient veto of muons and muon-related backgrounds [2].

\section{Oscillation Analysis}

The electron antineutrinos have a distinctive inverse beta decay (IBD) signature in the Daya Bay ADs:

$$
\bar{v}_{e}+p \rightarrow e^{+}+n
$$

The coincidence of the prompt scintillation from the positron and the delayed neutron capture on $\mathrm{Gd}$ (or $\mathrm{H}$ ) allows for powerful background rejection. For gadolinium-captured neutron events, we allow a subsequent single neutron signal with energy from 6 to $12 \mathrm{MeV}$ in a time window between 1 to $200 \mu$ s after the initial positron signal with energy from 0.7 to $12 \mathrm{MeV}$. Events with preceding cosmic muons or flashers [3] from the photomultiplier tubes are also rejected. We report our full set of cuts for the IBD selection in Ref. [3].

To further reduce uncertainties in the absolute energy calibration, an additional Flash-ADC readout system was installed in one $\mathrm{AD}$, and a special calibration campaign which used different radioactive source enclosures was performed. Therefore, in the most recent oscillation analysis, this uncertainty was controlled to less than $0.5 \%$ for visible energies larger than $2 \mathrm{MeV}$. The uncertainty for the cosmogenic ${ }^{9} \mathrm{Li}$ and ${ }^{8} \mathrm{He}$ background was reduced from $45 \%$ to $30 \%$ for the near detectors. We also studied the spent nuclear fuel history, which improved its uncertainty from $100 \%$ to $30 \%$. With 1958 days of data collection, more than 3.9 million antineutrino candidates were observed by all ADs, of which about 0.5 million were observed in the far site detectors. The new dataset provides a more than $60 \%$ increase of statistics from our previous publication [3].

With reduced systematic and statistical uncertainties, a $\chi^{2}$ expression was defined to compare the observation in the far-hall detectors to the prediction based on near-hall detectors measurement. The systematic variation was evaluated using simulation, and statistical variation was analytically estimated; both incorporated into the covariance matrix method (described as "Method A" in Ref. [3]).

Fig. 1 shows the background-subtracted reconstructed prompt energy spectrum at the far site, with the expectations derived from near-site measurements. Fig. 2 shows the fitted 2D contours regarding the $68.3 \%, 95.5 \%$, and $99.7 \%$ C.L. allowed regions. Our latest analysis yields $\sin ^{2} 2 \theta_{13}=0.0856 \pm 0.0029$ and $\Delta m_{32}^{2}=\left(2.471_{-0.070}^{+0.068}\right) \times 10^{-3} \mathrm{eV}^{2}$ assuming the normal hierarchy, 


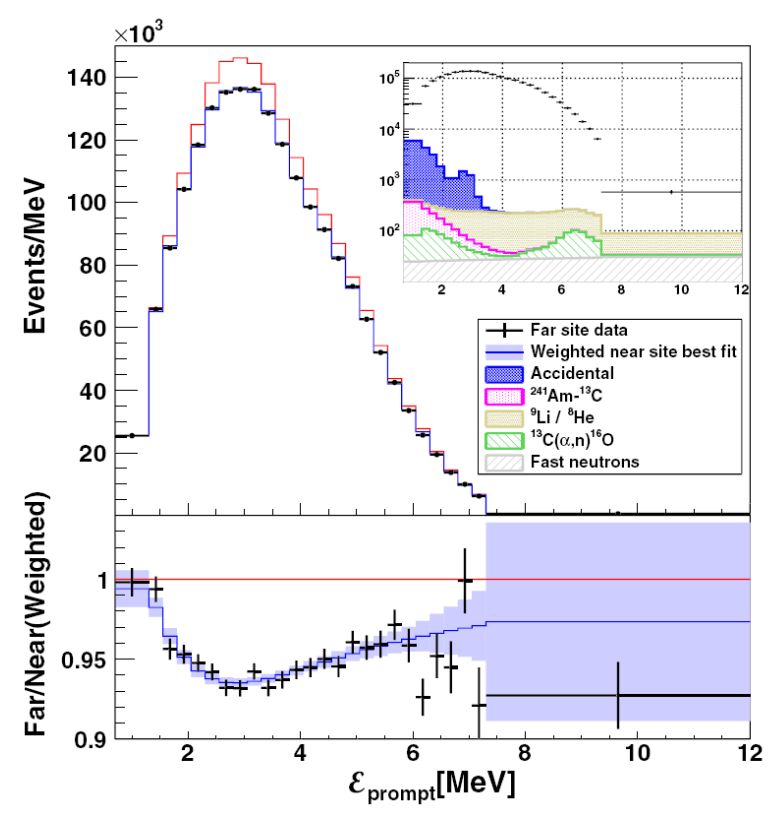

Figure 1: The reconstructed spectrum at the far site after background subtraction (black points) and the expectation derived from near-site measurements excluding (red line) and including (blue line) the best-fit oscillation. The ratio of measured data over predictions with no oscillation is shown at the bottom. The top right inset shows the background components with a logarithmic scale. Figure from Ref. [4].

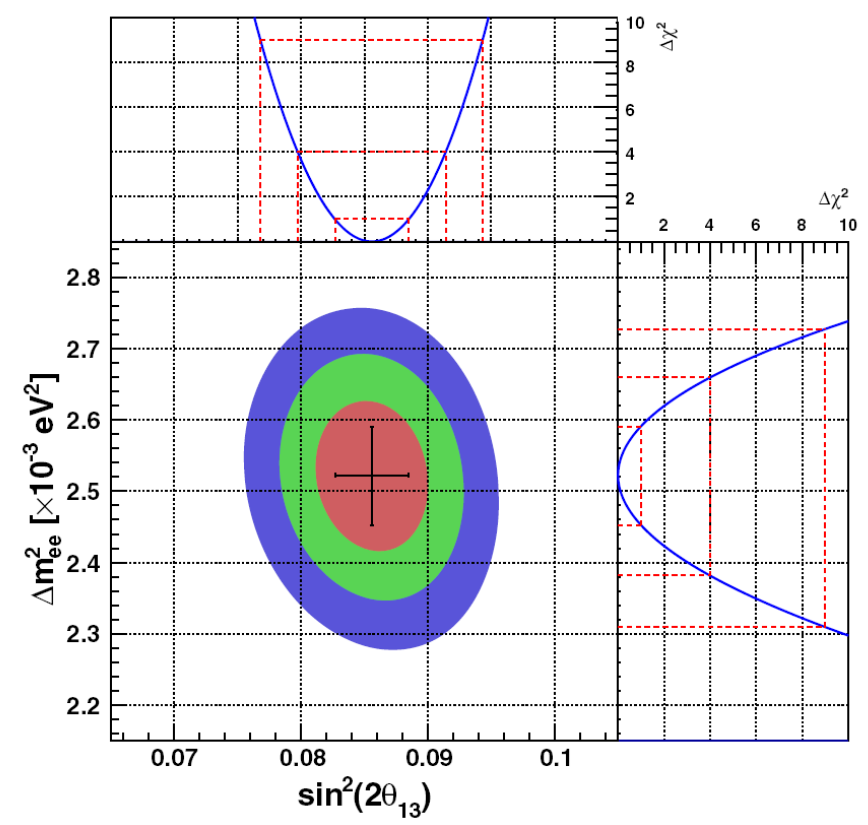

Figure 2: The $68.3 \%, 95.5 \%$, and $99.7 \%$ C.L. allowed regions in the $\Delta m_{e e^{2}}^{2} \sin ^{2} 2 \theta_{13}$ plane. The onedimensional plots represent the $\Delta \chi^{2}$ for $\sin ^{2} 2 \theta_{13}$ and $\Delta m_{e e}^{2}$ respectively. Figure from Ref .[4] 
and $\Delta m_{32}^{2}=-\left(2.575_{-0.070}^{+0.068}\right) \times 10^{-3} \mathrm{eV}^{2}$ assuming the inverted hierarchy. This is by far the most precise measurement of $\sin ^{2} 2 \theta_{13}$. The measurement of $\Delta m_{32}^{2}$ has comparable precision to that of the accelerator-based experiments $[5,6,7]$.

\section{References}

[1] An, F. P., et al. "The detector system of the Daya Bay reactor neutrino experiment." Nuclear Instruments and Methods in Physics Research Section A: Accelerators, Spectrometers, Detectors and Associated Equipment 811 (2016): 133-161.

[2] An, F. P., et al. "The muon system of the Daya Bay Reactor antineutrino experiment." Nuclear Instruments and Methods in Physics Research Section A: Accelerators, Spectrometers, Detectors and Associated Equipment 773 (2015): 8-20.

[3] An, Feng Peng, et al. "Measurement of electron antineutrino oscillation based on 1230 days of operation of the Daya Bay experiment." Physical Review D 95.7 (2017): 072006.

[4] Adey, D., et al. "Measurement of the Electron Antineutrino Oscillation with 1958 Days of Operation at Daya Bay." Physical Review Letters 121.24 (2018): 241805.

[5] Adamson, P., et al. "Combined analysis of $v_{\mu}$ disappearance and $v_{\mu} \rightarrow v_{e}$ appearance in MINOS using accelerator and atmospheric neutrinos." Physical review letters 112.19 (2014): 191801.

[6] Acero, M. A., et al. "New constraints on oscillation parameters from $v_{e}$ appearance and $v_{\mu}$ disappearance in the NOvA experiment." Physical Review D 98.3 (2018): 032012.

[7] Abe, K., et al. "Search for C P Violation in Neutrino and Antineutrino Oscillations by the T2K Experiment with $2.2 \times 10^{21}$ Protons on Target." Physical review letters 121.17 (2018): 171802 . 